\title{
Fast Ion Heating in Transient Collisionless Magnetic Reconnection via an Intrinsic Stochastic Mechanism
}

\author{
Young Dae Yoon (i) and Paul M. Bellan (1) \\ Department of Applied Physics and Materials Science California Institute of Technology, Pasadena, CA 91125, USA; yyoon@caltech.edu \\ Received 2018 October 11; revised 2018 October 31; accepted 2018 November 13; published 2018 November 26
}

\begin{abstract}
Stochastic heating has been known to be a powerful ion heating mechanism in the solar wind, atmosphere, and flares. In this Letter, we show that stochastic ion heating is inherent to transient collisionless magnetic reconnection. The explanation exploits the connected nature of electron canonical vorticity to show analytically that the in-plane electric and magnetic fields in a typical reconnection geometry satisfy the condition for stochastic heating of ions. Electron fluid simulations, test ion simulations, and comparisons to experiments all support the existence of this mechanism.
\end{abstract}

Key words: magnetic reconnection - plasmas - Sun: corona - Sun: flares - Sun: magnetic fields

\section{Introduction}

Magnetic reconnection is believed to be associated with anomalously strong ion energization and heating commonly observed in the solar corona, solar flares, and nanoflares (Priest et al. 1998; Sturrock 1999; Lin et al. 2003; Emslie et al. 2004). Evidence for anomalous ion energization and heating is abundant in magnetospheric (Sonnerup et al. 1981; Deng \& Matsumoto 2001), solar (Zhao et al. 2018), and laboratory (Hsu et al. 2000; Yoo et al. 2013; Chai et al. 2016; Hare et al. 2017) reconnection situations, suggesting that strong ion energization and heating is a fundamental property of reconnection. It is generally agreed that the microphysics underlying reconnection in these situations is collisionless regardless of the macrophysics (e.g., turbulence; Lazarian \& Vishniac 1999) that couples to the microscale. Because reconnection is believed to be collisionless and responsible for anomalous ion energization and heating, the specific mechanism by which reconnection causes ion energization and heating is expected to be collisionless.

Of the mechanisms that have been explored so far, stochastic ion heating (McChesney et al. 1987) is an enticing candidate because it is consistent with observed temperature anisotropy $\left(T_{\perp}>T_{\|}\right)$(Li et al. 1998; Cranmer \& van Ballegooijen 2003), strong solar atmosphere electric fields (Zhang \& Smartt 1986), and because many mechanisms can cause stochasticity. For example, Vranjes \& Poedts (2009a, 2009b, 2009c, 2010) have proposed stochastic heating driven by drift waves to be an important heating mechanism in the solar corona, while Chandran et al. (2010), Bourouaine \& Chandran (2013), Chandran et al. (2013), Stasiewicz et al. (2013), and Van Der Holst et al. (2014) have proposed that stochastic heating driven by Alfvén waves occurs in both the solar wind and the solar corona. However, to date stochastic ion heating has not been considered to be an intrinsic property of collisionless magnetic reconnection.

We show in this Letter that stochastic ion heating is indeed intrinsic to transient collisionless magnetic reconnection because stochastic ion heating will always occur at length scales smaller than the ion skin depth $d_{i}=c / \omega_{p i}$ when there is a perturbation of a Harris-type magnetic equilibrium (Harris 1962; Birn et al. 2001). Specifically, we show via analytic methods supplemented by numerical calculations that the in- plane electric fields near the magnetic separatrix satisfy the condition for stochastic ion heating. Comparisons to laboratory experiments support the analytical calculations presented here. These electric fields have been attributed to ion energization seen in previous numerical (Drake et al. 2009; Pritchett 2010; Aunai et al. 2011), observational (Wygant et al. 2005), and experimental (Stark et al. 2005; Yoo et al. 2013) reconnection studies, but the specific mechanism linking the fields to the heating has not been identified.

\section{Basic Equations and Setting}

Stochastic heating occurs when a particle in a magnetic field is subject to a time-dependent perpendicular potential gradient so strong that it causes a breakdown of the guiding-center approximation (McChesney et al. 1987; Stasiewicz et al. 2000; Bellan 2008, 2016). The particle then experiences a large potential difference within an orbital period and its motion becomes chaotic. When this happens to a large number of particles, the velocity distribution function broadens and this broadening is observationally equivalent to heating. The threshold in Coulomb gauge for ion stochastic heating is (McChesney et al. 1987; Stasiewicz et al. 2000; Bellan 2016)

$$
\frac{m_{i}}{q_{i} B^{2}}\left|\nabla_{\perp}^{2} \phi\right|=\frac{m_{i}}{q_{i} B^{2}}\left|\nabla_{\perp} \cdot \boldsymbol{E}_{\perp}\right|>1,
$$

where $\phi$ is the electrostatic potential, and $\nabla_{\perp}$ is the gradient perpendicular to $\boldsymbol{B}$.

Stochastic heating does not require the fields to be incoherent-it is a mechanism that causes particle trajectories to become chaotic in coherent fields. Thus stochastic heating should not be confused with mechanisms where the fields themselves are stochastic. It should be noted, however, that there has been extensive work on ion acceleration via stochastic interaction with magnetic islands (Hoshino 2012; Drake et al. 2013; Zank et al. 2014, 2015; Guo et al. 2015; Le Roux et al. 2015; le Roux et al. 2016). This Letter shows that stochastic ion heating occurs at a single $x$-line, but it will also work in the context of stochastic fields with multiple $\mathrm{x}$-lines if the condition in Equation (1) remains satisfied.

We begin the analysis by noting that the electric field acting on the ions can be determined from the two-fluid electron equation of motion. Using this equation to prescribe the electric 
field is equivalent to invoking the generalized Ohm's law with the Hall, electron inertia, and electron pressure terms all included. The magnitude of the magnetic field far from the reconnection region is denoted $B_{0}$ and this defines a reference electron cyclotron frequency $\omega_{c e}=q_{e} B_{0} / m_{e}$. On normalizing length by the electron collisionless skin depth $d_{e}=c / \omega_{p e}$, time by $\left|\omega_{c e}\right|^{-1}$, magnetic field by $B_{0}$, density by the background density $n_{0}$, electric field by $d_{e}\left|\omega_{c e}\right| B_{0}$, and pressure by $B_{0}^{2} / \mu_{0}$, the two-fluid electron equation of motion can be expressed as

$$
\overline{\boldsymbol{E}}+\overline{\boldsymbol{u}}_{e} \times \overline{\boldsymbol{B}}=-\frac{D \overline{\boldsymbol{u}}_{e}}{D \bar{t}}-\frac{\bar{\nabla} \bar{p}_{e}}{\bar{n}_{e}},
$$

where barred quantities are dimensionless, and $D / D \bar{t}=$ $\partial / \partial \bar{t}+\overline{\boldsymbol{u}}_{e} \cdot \bar{\nabla}$ is the convective derivative. This normalization is consistent with Faraday's law, i.e., $\bar{\nabla} \times \overline{\boldsymbol{E}}=-\partial \overline{\boldsymbol{B}} / \partial \bar{t}$. To reduce clutter, the bars will be dropped from now on. The normalized form of Equation (1) becomes

$$
\frac{1}{\varepsilon B^{2}}\left|\nabla_{\perp}^{2} \phi\right|>1,
$$

where $\varepsilon=m_{e} / m_{i}$ is the electron-to-ion mass ratio.

Taking the curl of Equation (2), assuming a negligible pressure term by positing either cold electrons or barotropic pressure, and invoking Faraday's law, gives the electron canonical induction equation (Kingsep et al. 1990; Bellan 2000)

$$
\frac{\partial \boldsymbol{Q}_{e}}{\partial t}=\nabla \times\left(\boldsymbol{u}_{e} \times \boldsymbol{Q}_{e}\right),
$$

where $\boldsymbol{Q}_{e} \equiv \nabla \times \boldsymbol{u}_{e}-\boldsymbol{B}$ is the electron canonical vorticity (Bellan 2000; You 2014). An important consequence of Equation (4) is that $\boldsymbol{Q}_{e}$ field lines remain connected, whereas $\boldsymbol{B}$ field lines may reconnect (Biskamp et al. 1997; Cafaro et al. 1998; Yoon \& Bellan 2017, 2018). Equivalently, Equation (4) means that the flux associated with $\boldsymbol{Q}_{e}$ is frozen into the moving electron fluid even though the flux associated with $\boldsymbol{B}$ is not. As noted in Yoon \& Bellan $(2017,2018)$, this distinction provides an extremely useful property for analyzing the internal details of collisionless magnetic reconnection.

It should be noted that in collisionless situations, pressure anisotropy is important (Egedal et al. 2013) and will add an additional term to Equation (4), allowing for the reconnection of $\boldsymbol{Q}_{e}$. However, this anisotropy effect will be disregarded in the present analysis because preliminary simulations that include this effect show that the resulting reconnection of $\boldsymbol{Q}_{e}$ is far slower than the reconnection of $\boldsymbol{B}$. Omitting pressure anisotropy keeps the analysis simple and enables us to focus on more dominant effects. Also for simplicity, a zero guide-field will be assumed, but it will be shown later that the analysis is valid in the inflow direction up to moderate guide fields and in the outflow direction up to strong guide fields.

By decomposing the convective derivative using $\boldsymbol{u}_{e} \cdot \nabla \boldsymbol{u}_{e}=\nabla u_{e}^{2} / 2-\boldsymbol{u}_{e} \times \boldsymbol{\nabla} \times \boldsymbol{u}_{e}$, Equation (2) may be expressed in terms of $\boldsymbol{Q}_{e}$ rather than $\boldsymbol{B}$, i.e.,

$$
\boldsymbol{E}=-\frac{\partial \boldsymbol{u}_{e}}{\partial t}+\boldsymbol{u}_{e} \times \boldsymbol{Q}_{e}-\nabla\left(\frac{u_{e}^{2}}{2}\right)-\frac{\nabla p_{e}}{n_{e}} .
$$

The connected nature of $\boldsymbol{Q}_{e}$ will be now used to simplify Equation (5) in a way that enables checking whether or not the condition given by Equation (1) for stochastic ion heating is satisfied in transient reconnection situations. The cold electron assumption will be used so that the hydrodynamic pressure term in Equation (5) may be ignored; justification for this assumption will be given later.

To validate the predictions of the model presented here, Equation (4) and other relevant equations have been numerically solved in the electron-magnetohydrodynamic (EMHD) regime for a perturbed 2D Harris-type current sheet configuration-details are described in Yoon \& Bellan $(2017,2018)$. The simulation gives the temporal evolution of $\boldsymbol{u}_{e}, \boldsymbol{Q}_{e}$, and $\boldsymbol{B}$ for times from $t=0$ to $t=580\left|\omega_{c e}\right|^{-1}$ in the domain $(x, y)=(200,600)=\left(30 d_{e}, 120 d_{e}\right)$. The origin is an X-point, there is inflow in the $\pm x$-directions, outflow in the $\pm y$ directions, and the system is observed to grow exponentially (Yoon \& Bellan 2017).

Figure 1(a) shows the numerically calculated reconnected inplane $\boldsymbol{B}$ field lines (white) and the connected in-plane $\boldsymbol{Q}_{e}$ field lines (red). The distinction between the reconnection of $\boldsymbol{B}$ and the non-reconnection of $\boldsymbol{Q}_{e}$ is clearly evident. Specifically, $B_{x}(x=0)$ is finite but $Q_{e x}(x=0)=0$, so that $\boldsymbol{B}$ reconnects but $\boldsymbol{Q}_{e}$ remains connected. The electric field is calculated numerically using the values of $\boldsymbol{u}_{e}$ and $\boldsymbol{Q}_{e}$ on the right-hand side (RHS) of Equation (5), and the associated electrostatic potential (color) is plotted for reference. This calculation is equivalent to keeping the Hall and the electron inertia contributions to the electric field $\left(\boldsymbol{u}_{e} \times \boldsymbol{B}\right.$ and $\left.D \boldsymbol{u}_{e} / D t\right)$ in Equation (2). The potential is saddle shaped with contours approximately following the $\boldsymbol{B}$ field lines in agreement with both previous simulations (Karimabadi et al. 2007; Drake et al. 2009; Pritchett 2010) and laboratory experiments (Yoo et al. 2013).

Yoon \& Bellan (2017) showed that the nominal growth rate of this system near the electron diffusion region (EDR) defined as $|x|<d_{e}$ can be expressed in terms of gradients of components of the electron flow. It is seen from the definition of $\boldsymbol{Q}_{e}$ that $\nabla \cdot \boldsymbol{Q}_{e}=0$ and it is also seen that $\nabla \cdot \boldsymbol{u}_{e} \sim \nabla \cdot \boldsymbol{J} \sim \nabla \cdot \nabla \times \boldsymbol{B}=0$. Using these properties and $Q_{e x}(x=0)=0$, expansion of the $y$-component of Equation (4) gives an exponentially growing solution for $Q_{e y}$ with a growth rate (Yoon \& Bellan 2017, Section IIIB)

$$
\frac{\partial}{\partial t} \simeq \frac{\partial u_{e y}}{\partial y} \simeq-\frac{\partial u_{e x}}{\partial x} .
$$

The growth rate is always positive because $\partial u_{e y} / \partial y \simeq \partial^{2} B_{z} / \partial x \partial y$ and the quadrupole out-of-plane Hall magnetic field has the dependence $B_{z} \sim x y$ in the EDR (Yoon $\&$ Bellan 2017). This growth rate will be used to rewrite the $\partial \boldsymbol{u}_{e} / \partial t$ term in Equation (5).

\section{Stochastic Heating Condition Analysis}

We first consider the inflow (i.e., flow on $y=0$, black dashed line in Figure 1(a)). The $\boldsymbol{B}$ field is in the $y$-direction here, so the perpendicular electric field relevant to stochastic heating is $E_{x}$. Although $E_{z}$ is also perpendicular to $B_{y}$, we need not consider $E_{z}$ for the purposes of analyzing stochastic heating because $\partial / \partial z=0$ in $2 \mathrm{D}$, so $E_{z}$ is irrelevant to Equation (1). Using the cold electron assumption (to be justified later), the $x$-component 
(a)

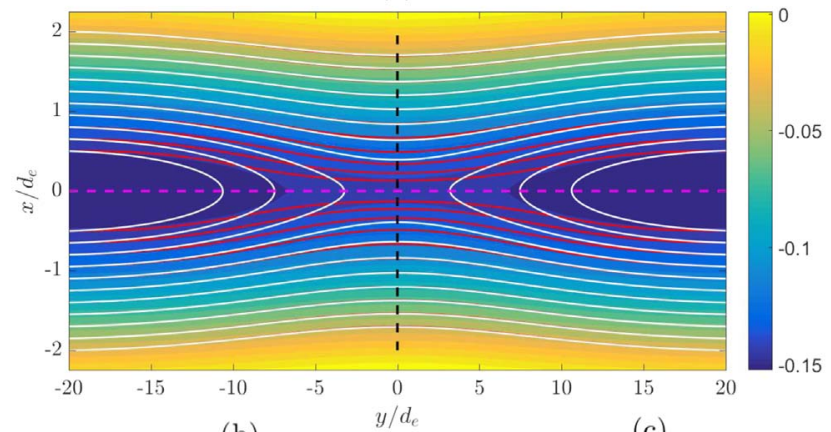

(b)

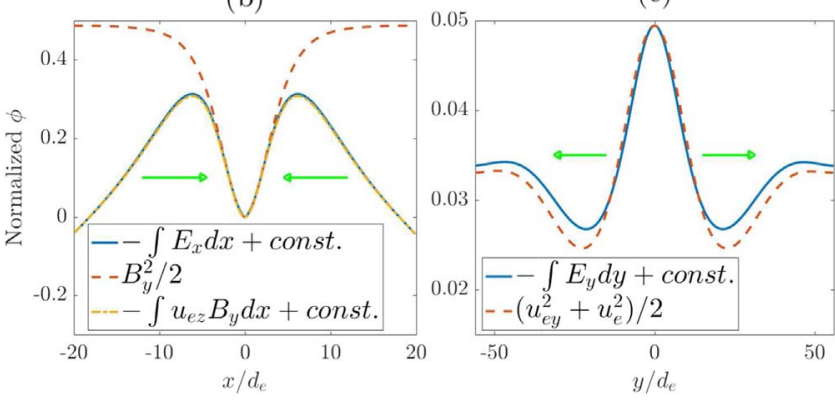

Figure 1. (a) Reconnected in-plane $\boldsymbol{B}$ field lines (white) and connected in-plane $\boldsymbol{Q}_{e}$ field lines (red) in the reconnection geometry. The effective potential (color) was calculated from the cold version of Equation (5). The inflows and outflows are, respectively, in the $\pm x$ - and $\pm y$-directions. The $x$-axis and the $y$-axis have different scales to show the field lines more evidently. (b) Comparison of $B_{y}^{2} / 2$ with $-\int E_{x} d x$ and $-\int u_{e z} B_{y} d x$ along the black dotted line in (a). (c) Comparison of $\left(u_{e y}^{2}+u_{e}^{2}\right) / 2$ with $-\int E_{y} d y$ along the magenta line in (a).

of Equation (5) becomes

$$
E_{x}=-\frac{\partial u_{e x}}{\partial t}+u_{e y} Q_{e z}-u_{e z} Q_{e y}-\frac{\partial}{\partial x}\left(\frac{u_{e}^{2}}{2}\right) .
$$

On invoking Equation (6), the first term on the RHS of Equation (7) is $-\partial u_{e x} / \partial t \simeq u_{e x} \partial u_{e x} / \partial x=\partial\left(u_{e x}^{2} / 2\right) / \partial x$. The second term disappears because $u_{e y}(y=0)=Q_{e z}(y=0)=0$ due to the antisymmetry of the outflow and the quadrupole nature (Yoon \& Bellan 2018) of the out-of-plane $\boldsymbol{Q}_{e}$ fields. On expanding the third term using $Q_{e y}=-\partial u_{e z} / \partial x-B_{y}$ and the fourth term using $u_{e}^{2}=u_{e x}^{2}+u_{e z}^{2}$ at $y=0$, Equation (7) simplifies to

$$
\begin{gathered}
E_{x}=u_{e z} B_{y} \\
\simeq-\frac{\partial}{\partial x}\left(\frac{B_{y}^{2}}{2}\right) .
\end{gathered}
$$

In the last line, we have used $\boldsymbol{u}_{e} \simeq-\nabla \times \boldsymbol{B}$ so $u_{e z}=\partial B_{x} / \partial y-\partial B_{y} / \partial x$ and $\partial B_{y} / \partial x \gg \partial B_{x} / \partial y$ in the EDR; these assumptions are true because $B_{y}>B_{x}$ and also $\partial / \partial x \gg \partial / \partial y$. Therefore, $B_{y}^{2} / 2$ acts as an effective electrostatic potential in the $x$-direction (this is equivalent to Equation (3) in Li \& Horiuchi 2008). This conclusion regarding the effective potential is shown graphically in Figure 1(b), where $-\int E_{x} d x$ and $-\int u_{e z} B_{y} d x$ are compared with $B_{y}^{2} / 2$, calculated from the simulation. Outside of the EDR $\partial B_{y} / \partial x \longrightarrow 0$, so the approximation fails, but for the purposes of analyzing the slope of the potential valley into which the ions fall (ion inflow directions represented by the green arrows) and thus for the purpose of investigating the existence of stochastic heating, $B_{y}^{2} / 2$ remains a good approximation for the electrostatic potential. The integration constant was set so that the three functions coincide at $x=0$ in Figure 1(b).

Equation (3), the condition for ion stochasticity in the inflow direction, can thus be written as

$$
\frac{1}{\epsilon B_{y}^{2}}\left|\frac{\partial^{2}}{\partial x^{2}}\left(\frac{B_{y}^{2}}{2}\right)\right|>1
$$

Using $\quad B_{y}(x) \simeq B_{0} x / L_{x} \quad$ for $\quad x<L_{x}$ and $d_{e}^{2} / \varepsilon=d_{i}^{2}$, Equation (10) in dimensioned quantities becomes $x^{2}<d_{i}^{2}$. A sufficient condition to satisfy $x^{2}<d_{i}^{2}$ while $x<L_{x}$ is

$$
L_{x}^{2}<d_{i}^{2}
$$

Therefore, at sub-ion-skin-depth length scales, at which collisionless reconnection occurs, the condition for stochastic heating in the inflow direction is satisfied.

The cold electron assumption in the inflow direction and thus the validity of Equation (9) will now be justified. Equation (9) can be obtained directly from Harris's exact kinetic current sheet solution (Harris 1962) derived in a frame moving at $\boldsymbol{V}=\left(\boldsymbol{u}_{i}+\boldsymbol{u}_{e}\right) / 2$, i.e., at the average of the electron and ion mean velocities and recalling that Harris showed that the electrostatic potential is exactly zero in this frame. Taylor expanding for small $x$ the magnetic field $\boldsymbol{B}_{\text {Harris }}$ given by Equation (20) in Harris (1962), Lorentz transforming back to the lab frame so that $\boldsymbol{E}_{\text {lab }}=\boldsymbol{V} \times \boldsymbol{B}_{\text {Harris }}$, and integrating to find the lab-frame electrostatic potential yields Equation (9) here. Also, numerical (Li \& Horiuchi 2008) and experimental (Yoo et al. 2013) studies show that the magnetic force on the electron fluid is balanced by the electric field corresponding to Equation (8) and thus Equation (9).

We now consider the outflow (flow along the $x=0$ line, magenta dashed line in Figure 1(a)). Because the magnitude of $\boldsymbol{B}$ is very small on this line, the cold electron assumption in principle cannot be used. However, it will be shown later that including the electron pressure term has little effect on the stochasticity condition, so for now electrons will be assumed cold. Along the outflow (i.e., on the $y$-axis, $x=0$ ), the magnetic field is in the $x$-direction, so the perpendicular electric field relevant to stochastic heating is $E_{y}$. The magnetic field component $B_{x}$ at $x=0$ has small amplitude and its being finite is what constitutes the reconnection. $E_{z}$ again plays no role in Equation (1) because $\partial / \partial z=0$. The $y$-component of Equation (5) with the cold electron assumption invoked is

$$
E_{y}=-\frac{\partial u_{e y}}{\partial t}+u_{e z} Q_{e x}-u_{e x} Q_{e z}-\frac{\partial}{\partial y}\left(\frac{u_{e}^{2}}{2}\right) \text {. }
$$

Using Equation (6), the first term on the RHS of Equation (12) is $-\partial u_{e y} / \partial t \simeq-u_{e y} \partial u_{e y} / \partial y=-\partial\left(u_{e y}^{2} / 2\right) / \partial y$. The second term disappears because $Q_{e x}(x=0)=0$ as $Q_{e}$ does not reconnect. The third term disappears because of the antisymmetry of $u_{e x}$ and the quadrupole nature of $Q_{e z}$. Equation (12) now simplifies to

$$
E_{y} \simeq-\frac{\partial}{\partial y}\left(\frac{u_{e y}^{2}+u_{e}^{2}}{2}\right)
$$

Thus, $\left(u_{e y}^{2}+u_{e}^{2}\right) / 2$ acts as an effective potential in the $y$ direction. A comparison between $-\int E_{y} d x$ and $\left(u_{e y}^{2}+u_{e}^{2}\right) / 2$ along $x=0$ shows that the latter is a good approximation, as 
shown in Figure 1(c). The potential forms a hill off of which the ions fall downward (ion outflow directions represented by green arrows). The integration constant was set in Figure 1(c) so that the two functions coincide at $y=0$. The normalized stochasticity condition Equation (3) in the outflow direction is

$$
\frac{1}{\varepsilon B_{x}^{2}}\left|\frac{\partial^{2}}{\partial y^{2}}\left(\frac{u_{e y}^{2}+u_{e}^{2}}{2}\right)\right|>1 .
$$

Here the connected nature of $\boldsymbol{Q}_{e}$ gives $Q_{e x}(x=0)=$ $\partial u_{e z} / \partial y-B_{x} \simeq u_{e z} / L_{y}-B_{x}=0$, so using $\partial / \partial y \sim 1 / L_{y}=$ $B_{x} / u_{e z}$ on Equation (14) gives

$$
\frac{1}{\varepsilon}\left(\frac{u_{e y}^{2}+u_{e}^{2}}{2 u_{e z}^{2}}\right)>1 \text {. }
$$

However, $\quad\left(u_{e y}^{2}+u_{e}^{2}\right) / u_{e z}^{2} \geqslant 1 \quad$ as $\quad u_{e}^{2}=u_{e y}^{2}+u_{e z}^{2}$,

Equation (15) reduces to

$$
\frac{m_{i}}{m_{e}}>1,
$$

which is of course satisfied. Consequently, the stochasticity condition in the outflow direction is always satisfied by a wide margin.

The cold electron assumption in the outflow direction will now be justified. Recalling that $\bar{p}_{e}=n_{e} k_{B} T_{e} /\left(B_{0}^{2} / \mu_{0}\right)$ gives $\bar{p}_{e}=\bar{n}_{e} \bar{v}_{T e}^{2} / 2$ where $\bar{v}_{T e}^{2}=\left(2 k_{B} T_{e} / m_{e}\right) /\left(d_{e}\left|\omega_{c e}\right|\right)^{2}$. Dropping bars, the pressure term in Equation (5) becomes $\frac{\nabla p_{e}}{n_{e}}=\nabla\left(\frac{v_{T e}^{2}}{2}\right)+\frac{v_{T e}^{2}}{2} \frac{\nabla n_{e}}{n_{e}} \simeq \nabla\left(\frac{v_{T e}^{2}}{2}\right)$. The $\frac{v_{T e}^{2}}{2} \frac{\nabla n_{e}}{n_{e}}$ term was dropped by invoking the quasi-neutrality condition; because the normalized Poisson's equation gives $\nabla \cdot \boldsymbol{E}=$ $-\frac{c^{2}}{v_{A e}^{2}}\left(n_{e}-n_{0}\right)$ where $v_{A e}^{2}=B^{2} / \mu_{0} m_{e} n_{e}$ is the electron Alfvén velocity, taking the gradient of both sides and requiring $\nabla \cdot \boldsymbol{E}$ to be small by quasi-neutrality yields $\nabla n_{e} \ll v_{A e}^{2} / c^{2}$. Using this new pressure term in Equation (5) and applying the same reasoning used to derive Equation (13), $E_{y}$ approximates to

$$
E_{y} \simeq-\frac{\partial}{\partial y}\left(\frac{u_{e y}^{2}+u_{e}^{2}+v_{T e}^{2}}{2}\right) .
$$

Equation (17) implies that if there is electron heating in the exhaust region, the potential becomes flatter and thus less conducive to stochastic heating. This is not a problem in guidefield reconnection in which electron heating is localized near the EDR (Tanabe et al. 2015). For zero guide-field reconnection, however, if the exhaust region (finite $y$ ) is hotter than the EDR $(y \approx 0$; Yamada et al. 2014), the potential is a well centered around $y=0$, which is not conducive to stochastic heating. Nevertheless, temperature effects do not negate the stochastic condition being satisfied for the following reasons.

Consider the worst-case scenario where the outflow becomes completely thermalized, i.e., $u_{e y}^{2}+v_{T e}^{2}=$ const. In this case, only $u_{e}^{2}$ is left in Equation (17) so Equation (15) becomes $\frac{1}{\varepsilon}\left(\frac{u_{e}^{2}}{2 u_{e z}^{2}}\right)>1$, which is always satisfied because $u_{e}^{2} \geqslant u_{e z}^{2}$.

Furthermore, previous studies are consistent with the cold electron assumption. Several numerical studies (Karimabadi et al. 2007; Pritchett 2010) have shown that $\left|e \Phi / T_{e}\right|$-which corresponds to $\sim u_{e}^{2} / v_{T e}^{2}$-increases to be $10 \sim 20$ inside of the saddle potential, which means that the electron flow is cold and laminar. A previous experimental study (Yoo et al. 2013) measured the plasma potential including electron temperature effects and showed that the potential is a hill in the $y$-direction with the peak at $y=0$, confirming the shape given in Figure 1(c).

In the presence of a guide-field $B_{z 0}$, the denominator of Equation (10) changes from $\epsilon B_{y}^{2}$ to $\epsilon\left(B_{y}^{2}+B_{z 0}^{2}\right)$. Thus in the inflow direction, the stochastic heating mechanism is valid up to guide-field strengths that are comparable to the shear field strength. In the outflow direction, the denominator of Equation (14) changes to $\epsilon\left(B_{x}^{2}+B_{z 0}^{2}\right)$, so the stochastic condition becomes $\frac{m_{i}}{m_{e}} \frac{B_{x}^{2}}{B_{x}^{2}+B_{z 0}^{2}}>1$. Because $\left|B_{x}\right|$ increases as reconnection proceeds and becomes of the same order as shear magnetic field $\left|B_{y}\right|$, the mechanism is valid in the outflow direction for strong guide fields up to $\frac{B_{z 0}^{2}}{B_{y}^{2}}<\frac{m_{i}}{m_{e}}$.

The fact that Equations (11) and (16) do not depend on the magnetic field signifies that stochastic heating is intrinsic to collisionless reconnection. $\phi$ in Equation (3) has a dependence on $\boldsymbol{B}$ in such a way that this dependence cancels out $\boldsymbol{B}$ in the denominator. Thus, Equation (3) is always met given that the assumptions in the present analysis are met as well.

\section{Test Particle Simulation}

In order to verify ion stochastic heating, 6000 test ion particle trajectories were simulated using an implicit particle integrator (Birdsall \& Langdon 2004). The trajectories were calculated for both stationary and growing electric fields. These two cases should give, respectively, ordered and stochastic ion motions. The particles were started at rest from a rectangular grid $(x, y)=(0,0)-(4.5,0.4) d_{e}$, spaced by $(\Delta x, \Delta y)=(0.015,0.02) d_{e}$. Such a small spatial separation was chosen because stochasticity is defined by particles' having disparate trajectories with slightly different initial conditions. The results presented in Figure 2 are based on a mass ratio $m_{i} / m_{e}=10$. While this mass ratio is small, the purpose of the simulation is to reveal the distinction between coherent and stochastic situations. Simulations with higher mass ratios (20, $50,100)$ have also been conducted and exhibit similar qualitative behavior; mass and time scalings are subject to future investigations.

The growing electric field was the time-dependent numerically calculated field, and the stationary electric field was chosen to have the value of the time-dependent field at $t=5\left|\omega_{c e}\right|^{-1}$. A small value for this reference time was chosen because the particles were started at rest from different positions. This means that they all have different starting energies, so a small potential was chosen to keep the ions initially cold in order to explicitly show the thermal energy gain due to stochastic heating. Coherence was not destroyed by choosing the electric field at a later time. The focus will be on the outflow direction because Equation (16) is met by a large margin so the stochastic evolution in this direction is more evident.

Figure 2(a) shows 10 selected ion trajectories (red) in the stationary electric field case, and Figure 2(e) shows the stochastic counterpart. The effective potential at (a) $t=5\left|\omega_{c e}\right|^{-1}$ and (e) $580\left|\omega_{c e}\right|^{-1}$ is plotted (color) for reference. The ions have Speiser-type orbits, consistent with previous numerical studies (Drake et al. 2009; Aunai et al. 2011). In the stationary electric field case (i.e., Figure 2(a)), the trajectories 
(a)

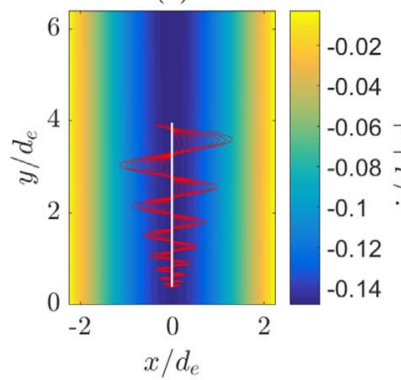

(e)

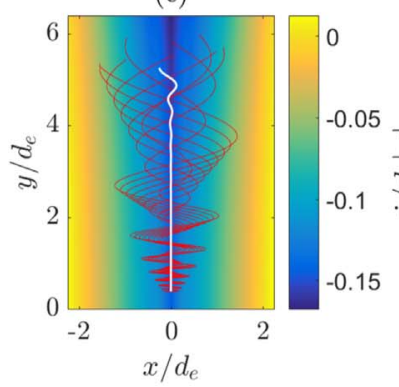

(b)

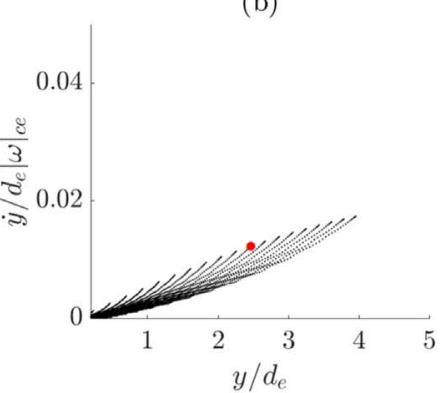

(f)

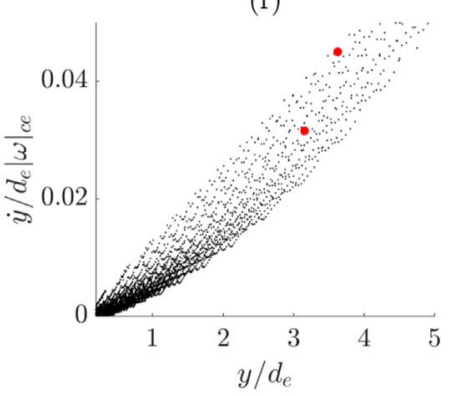

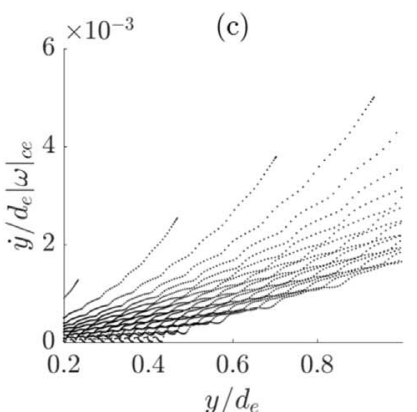

(g)

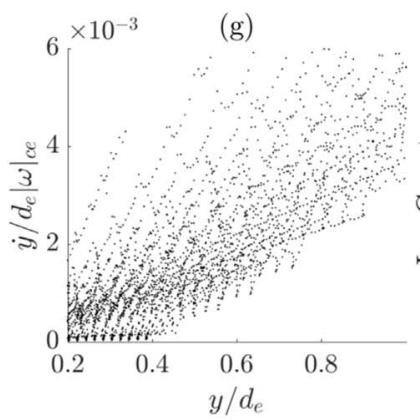

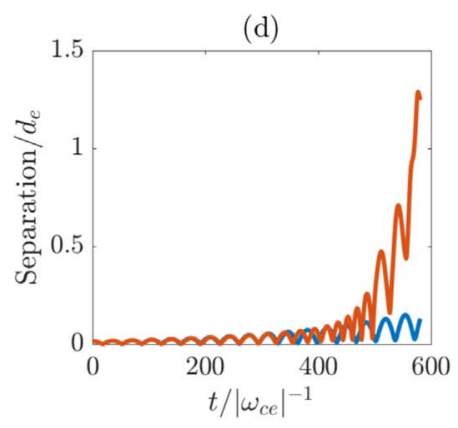

(h)

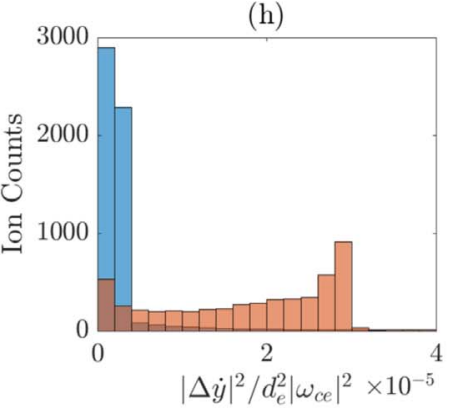

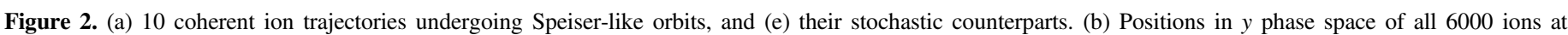

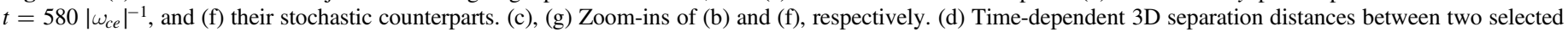

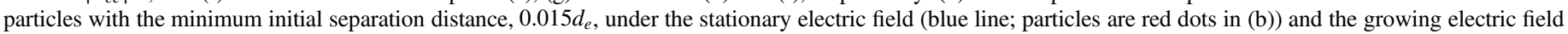
(red line; particles are red dots in (f)). (h) Distribution of $|\Delta \dot{y}|^{2}$ for the coherent case (blue) and the stochastic case (red).

show a coherent motion. In the growing electric field case (i.e., Figure 2(e)), the trajectories clearly diverge and undergo phase mixing, the signature of stochastic motion. Stochasticity is further demonstrated by the central trajectory (white), which starts at $x=0$ in both situations and which, in principle, should have no motion in the $x$-direction. While the central trajectory in Figure 2(a) (stationary electric field) is a straight, stable outflow, the trajectory in Figure 2(e) (growing electric field) becomes unstable indicating stochasticity, where the seed for the instability is the ever-present small numerical error.

Examination of the ion phase space indicates chaotic motion. Figure 2(b) shows the positions in $y$ phase space of all ions at $t=580\left|\omega_{c e}\right|^{-1}$ for the stationary electric field case, and Figure 2(f) shows the counterpart in the growing electric field case. Figures 2(c) and (g) show zoomed-in versions of Figures 2(b) and (f), respectively. The upper plots (stationary electric field) clearly show a periodic order, whereas the lower plots (growing electric field) exhibit unpredictability and chaos.

The two red dots in Figure 2(b) (which look like one dot due to their proximity) represent the phase space positions of two selected particles that started with the minimal separation of $0.015 d_{e}$ for the stationary electric field case, and those in Figure 2(f) represent their stochastic counterpart (growing electric field). The time-dependent 3D separation distance is plotted in Figure 2(d) as blue (ordered) and red (stochastic). It can be seen in both phase and physical space that the two particles in the stochastic case separate from one another exponentially, a behavior that is the characteristic of chaotic motion and in accordance with the concept of Lyapunov exponents (Lyapunov 1992).

The stochasticity results in an effective heating. Figure 2(h) shows for ordered (blue) and stochastic (red) ions the distribution of the squared deviation of the ion outflow velocity $|\Delta \dot{y}|^{2}$, a proxy for thermal energy. Significant heating is clearly seen for the stochastic case. The distribution is nonMaxwellian, however, because collisions are not included.
In reality, the ions start with an initial temperature. Another simulation was conducted where the ions start with a thermal velocity of $0.01 d_{e}\left|\omega_{c e}\right|$, and the results are shown in Figure 3. The phase space as shown in Figure 3(b) is much broader than that in Figure 3(a). The separation between the two red dots is again much larger in Figure 3(b) than in Figure 3(a). The thermal spread can be seen explicitly in Figure 3(d) in contrast to Figure 3(c). Also, in comparison with Figures 2(b) and (f), it can be seen that an initial temperature makes the ions spatially travel farther and faster.

It should be said that the purpose of this test particle simulation is to explicitly show the contrast between coherent and stochastic ion motion, and the results presented here reflect the initial stage of stochastic evolution. A temporal limitation exists due to the timescale separation between the ions and electrons, so the simulation must be halted before the ions are exhausted from the reconnection region. This also means that the ions have yet to gain all of the available energy from this mechanism.

\section{Discussion}

An order-of-magnitude estimate for the heating rate and strength in a reconnection situation will be given as follows. A proxy for the exponential heating rate from this mechanism in a reconnection situation is its Lyapunov exponent, which is $\gamma_{E}=\sqrt{\frac{q_{i} E}{m_{i} a}}$, where $a$ is the length scale of the electric field $E$ (Bellan 2016). Here, $\sqrt{\frac{q_{i} E}{m_{i} a}}=\frac{q_{i} B_{0}}{m_{i}} \frac{B_{\perp}}{B_{0}} \sqrt{\frac{m_{i} E}{q_{i} B_{\perp}^{2} a}}$ and noting that the quantity inside the square root is equivalent to the left-hand side (LHS) of the stochastic heating criterion (Equation (1)), $\gamma \approx \omega_{c i} \frac{B_{\perp}}{B_{0}} \frac{d_{i}}{L_{x}}$ for the inflow and $\approx \omega_{c i} \frac{B_{\perp}}{B_{0}} \sqrt{\frac{m_{i}}{m_{e}}}$ for the outflow. Assuming $B_{\perp} \lesssim B_{0}$ and $L_{x} \lesssim d_{i}$, the theoretical heating rate $\gamma$ is in the range $\omega_{c i}<\gamma<\sqrt{\omega_{c i} \omega_{c e}}$. This is much faster than the 

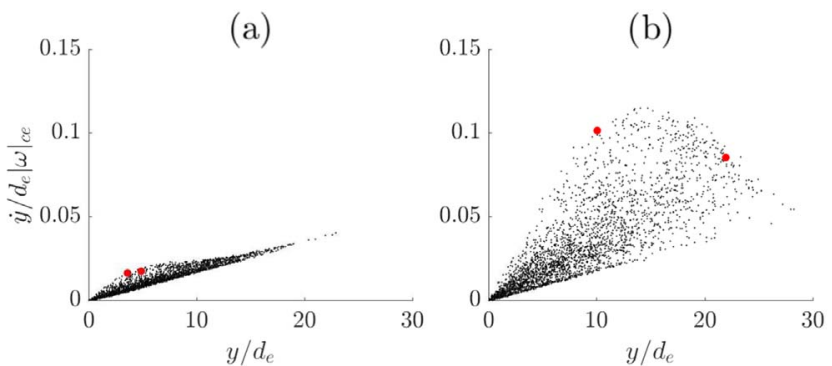

(c)
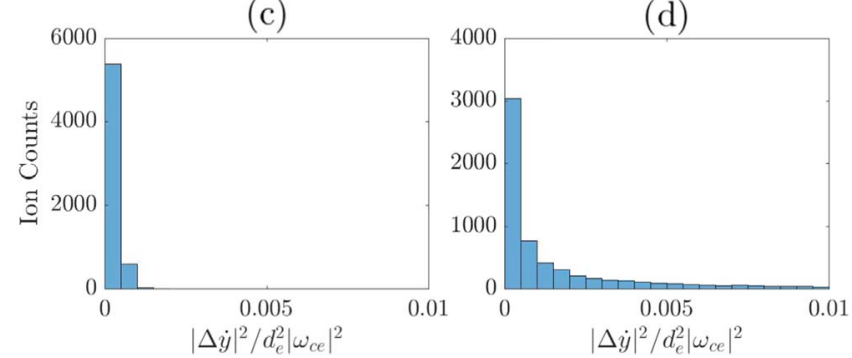

Figure 3. Positions in $y$ phase space of ions that started with a thermal velocity of $0.01 d_{e}\left|\omega_{c e}\right|$ in (a) the coherent case and (b) the stochastic case. Distribution of $|\Delta \dot{y}|^{2}$ in (c) the coherent case and (d) the stochastic case.

MHD timescale governing the macrophysics of the solar environment.

As noted in Sanders et al. (1998), the maximally achieved ion thermal velocity due to stochastic heating is $v_{T}=\omega_{c i} a(\alpha+1.9)$, where $\alpha$ is the LHS of Equation (1) and thus satisfies $\alpha>1$. Using $a \simeq d_{i}$, the upper bound on the local beta is $\beta_{\text {local }}=v_{T} / v_{A}=\alpha+1.9$, at a timescale faster than the ion cyclotron frequency. As the reconnected magnetic field magnitude is less than that of the upstream field, the global beta may still stay sub-unity. A more detailed analysis on the heating amount is subject to future investigations.

Our results are consistent with a recent particle-in-cell calculation of ion temperature profile in collisionless reconnection. Haggerty et al. (2015, their Figure 2) showed a sharp ion temperature increase across the magnetic separatrix where the potential drop occurs, and a further increase in the ion outflow jet. This is congruous with the fact that the inflow condition (Equation (11)) is marginally satisfied and the outflow condition (Equation (16)) is satisfied by a wide margin; therefore, the temperature increase is more dramatic along the outflow.

The claim that Equation (1) is satisfied in collisionless reconnection is also supported by experimental observations. In the Magnetic Reconnection eXperiment (MRX; Yamada et al. 2014), the measured in-plane electric field was $700 \mathrm{~V} \mathrm{~m}^{-1}$ with a $1 \mathrm{~cm}$ length scale. Using the upstream magnetic field $B_{0}=0.1 \mathrm{kG}$, Equation (1) becomes $14.6 \gg 1$ and so the condition for ion stochastic heating is satisfied by a wide margin. The condition was also met in the spontaneous collisionless reconnection event in the Caltech MHD-driven jet experiment (Chai et al. 2016).

The calculated heating rate also agrees with experiments. Defining $\gamma_{\text {obs }}$ to be the inverse e-folding timescale of the ion temperature based on experimental observations, $\gamma_{\text {obs }} \approx$ $10^{5} \mathrm{~s}^{-1}$ for MRX (Yamada et al. 2014) and $\gamma_{\mathrm{obs}} \approx 10^{6} \mathrm{~s}^{-1}$ for the Caltech jet experiment (Chai et al. 2016). Calculation of $\gamma$ range for both experiments gives $\gamma \approx 1-30 \times 10^{6} \mathrm{~s}^{-1}$ for the former and $\gamma \approx 1-100 \times 10^{6} \mathrm{~s}^{-1}$ for the latter. Therefore, stochastic ion heating is fast enough to account for the observations in both experiments.

\section{Conclusion}

It has been shown that ion stochastic heating, which had already been established as an important heating mechanism in the solar wind, atmosphere, and flares, is also inherent to transient collisionless magnetic reconnection. The connected nature of electron canonical vorticity has been exploited to show that the electric fields near the magnetic separatrix satisfy the stochastic heating criterion. The approximations for the electric field were verified by an electron fluid simulation, and ion stochasticity was demonstrated by test particle simulations. Comparisons to laboratory reconnection experiments also support the existence of this mechanism.

This material is based upon work supported by the U.S. Department of Energy Office of Science, Office of Fusion Energy Sciences under award No. DE-FG02-04ER54755 and by the Air Force Office of Scientific Research under award No. FA9550-17-1-0023.

\section{ORCID iDs}

Young Dae Yoon 주 https://orcid.org/0000-0001-8394-2076 Paul M. Bellan (1) https://orcid.org/0000-0002-0886-8782

\section{References}

Aunai, N., Belmont, G., \& Smets, R. 2011, JGRA, 116, A09232 Bellan, P. M. 2000, Spheromaks (Singapore: World Scientific) Bellan, P. M. 2008, Fundamentals of Plasma Physics (Cambridge: Cambridge Univ. Press)

Bellan, P. M. 2016, JPIPh, 82, 615820101

Birdsall, C. K., \& Langdon, A. B. 2004, Plasma Physics Via Computer Simulation (Boca Raton, FL: CRC Press)

Birn, J., Drake, J. F., Shay, M. A., et al. 2001, JGRA, 106, 3715

Biskamp, D., Schwarz, E., \& Drake, J. F. 1997, PhPl, 4, 1002

Bourouaine, S., \& Chandran, B. D. 2013, ApJ, 774, 96

Cafaro, E., Grasso, D., Pegoraro, F., Porcelli, F., \& Saluzzi, A. 1998, PhRvL, 80,4430

Chai, K.-B., Zhai, X., \& Bellan, P. M. 2016, PhPl, 23, 032122

Chandran, B. D., Verscharen, D., Quataert, E., et al. 2013, ApJ, 776, 45

Chandran, B. D. G., Li, B., Rogers, B. N., Quataert, E., \& Germaschewski, K. 2010, ApJ, 720, 503

Cranmer, S. R., \& van Ballegooijen, A. A. 2003, ApJ, 594, 573

Deng, X. H., \& Matsumoto, H. 2001, Natur, 410, 557

Drake, J. F., Swisdak, M., \& Fermo, R. 2013, ApJL, 763, 1

Drake, J. F., Swisdak, M., Phan, T. D., et al. 2009, JGRA, 114, A05111

Egedal, J., Le, A., \& Daughton, W. 2013, PhPl, 20, 061201

Emslie, A. G., Kucharek, H., Dennis, B. R., et al. 2004, JGRA, 109, A10104

Guo, F., Liu, Y. H., Daughton, W., \& Li, H. 2015, ApJ, 806, 167

Haggerty, C. C., Shay, M. A., Drake, J. F., Phan, T. D., \& McHugh, C. T. 2015, GeoRL, 42, 9657

Hare, J. D., Suttle, L., Lebedev, S. V., et al. 2017, PhRvL, 118, 085001

Harris, E. G. 1962, NCim (1955-1965), 23, 115

Hoshino, M. 2012, PhRvL, 108, 1

Hsu, S. C., Fiksel, G. A., Carter, T. A., et al. 2000, PhRvL, 84, 3859

Karimabadi, H., Daughton, W., \& Scudder, J. 2007, GeoRL, 34, L13104

Kingsep, A. S., Chukbar, K. V., \& Yan'kov, V. V. 1990, in Reviews of Plasma Physics, Vol. 16, ed. B. Kadomtsev (New York: Consultants Bureau), 243 Lazarian, A., \& Vishniac, E. T. 1999, ApJ, 517, 700

Le Roux, J. A., Zank, G. P., Webb, G. M., \& Khabarova, O. 2015, ApJ, 801, 1

le Roux, J. A., Zank, G. P., Webb, G. M., \& Khabarova, O. V. 2016, ApJ, 827,47

Li, B., \& Horiuchi, R. 2008, PhRvL, 101, 215001

Li, X., Habbal, S. R., Kohl, J., \& Noci, G. 1998, ApJL, 501, L133

Lin, R. P., Krucker, S., Hurford, G. J., et al. 2003, ApJL, 595, L69

Lyapunov, A. M. 1992, IJC, 55, 531

McChesney, J. M., Stern, R. A., \& Bellan, P. M. 1987, PhRvL, 59, 1436 
Priest, E. R., Foley, C. R., Heyvaerts, J., et al. 1998, Natur, 393, 545

Pritchett, P. L. 2010, JGRA, 115, A10208

Sanders, S. J., Bellan, P. M., \& Stern, R. A. 1998, PhPl, 5, 716

Sonnerup, B. U. Ö, Paschmann, G., Papamastorakis, I., et al. 1981, JGRA, 86, 10049

Stark, A., Fox, W., Egedal, J., Grulke, O., \& Klinger, T. 2005, PhRvL, 95, 235005

Stasiewicz, K., Lundin, R., \& Marklund, G. 2000, PhyS, 2000, 60

Stasiewicz, K., Markidis, S., Eliasson, B., Strumik, M., \& Yamauchi, M. 2013, EL, 102, 49001

Sturrock, P. A. 1999, ApJ, 521, 451

Tanabe, H., Yamada, T., Watanabe, T., et al. 2015, PhRvL, 115, 1

Van Der Holst, B., Sokolov, I. V., Meng, X., et al. 2014, ApJ, 782, 81

Vranjes, J., \& Poedts, S. 2009a, PhP1, 16, 092902
Vranjes, J., \& Poedts, S. 2009b, MNRAS, 400, 2147

Vranjes, J., \& Poedts, S. 2009c, MNRAS, 398, 918

Vranjes, J., \& Poedts, S. 2010, ApJ, 719, 1335

Wygant, J. R., Cattell, C. A., Lysak, R., et al. 2005, JGRA, 110, A09206

Yamada, M., Yoo, J., Jara-Almonte, J., et al. 2014, NatCo, 5, 4774

Yoo, J., Yamada, M., Ji, H., \& Myers, C. E. 2013, PhRvL, 110, 215007

Yoon, Y. D., \& Bellan, P. M. 2017, PhPl, 24, 052114

Yoon, Y. D., \& Bellan, P. M. 2018, PhPl, 25, 055704

You, S. 2014, PPCF, 56, 064007

Zank, G. P., Hunana, P., Mostafavi, P., et al. 2015, ApJ, 814, 137

Zank, G. P., Le Roux, J. A., Webb, G. M., Dosch, A., \& Khabarova, O. 2014, ApJ, 797, 28

Zhang, Z., \& Smartt, R. N. 1986, SoPh, 105, 355

Zhao, L.-L., Zank, G. P., Khabarova, O., et al. 2018, ApJL, 864, L34 